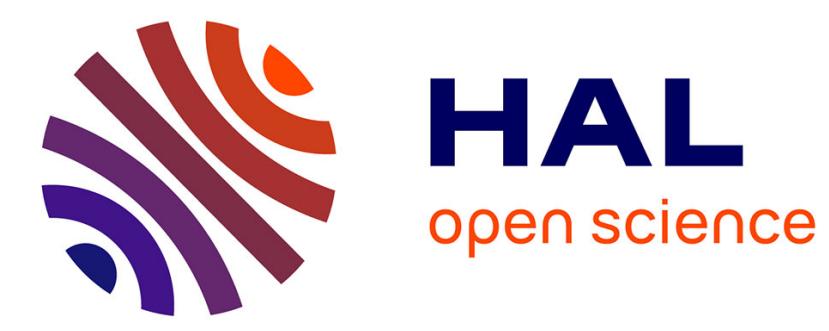

\title{
Underscreening in ionic liquids: a first principles analysis
} Benjamin Rotenberg, Olivier Bernard, Jean-Pierre Hansen

\section{To cite this version:}

Benjamin Rotenberg, Olivier Bernard, Jean-Pierre Hansen. Underscreening in ionic liquids: a first principles analysis. Journal of Physics: Condensed Matter, 2018, 30 (5), pp.054005. 10.1088/1361648X/aaa3ac . hal-01896764

\section{HAL Id: hal-01896764 https: / hal.sorbonne-universite.fr/hal-01896764}

Submitted on 16 Oct 2018

HAL is a multi-disciplinary open access archive for the deposit and dissemination of scientific research documents, whether they are published or not. The documents may come from teaching and research institutions in France or abroad, or from public or private research centers.
L'archive ouverte pluridisciplinaire HAL, est destinée au dépôt et à la diffusion de documents scientifiques de niveau recherche, publiés ou non, émanant des établissements d'enseignement et de recherche français ou étrangers, des laboratoires publics ou privés. 


\title{
Underscreening in ionic liquids: a first principles analysis
}

\author{
Benjamin Rotenberg $^{1,2}$, Olivier Bernard ${ }^{1}$, Jean-Pierre Hansen ${ }^{1,3}$ \\ ${ }^{1}$ Sorbonne Universités, UPMC Univ Paris 06, CNRS, Laboratoire PHENIX, Case \\ 51, 4 place Jussieu, F-75005 Paris, France \\ ${ }^{2}$ Réseau sur le Stockage Electrochimique de l'Energie (RS2E), FR CNRS 3459, \\ 80039 Amiens Cedex, France. \\ ${ }^{3}$ Department of Chemistry, University of Cambridge, Cambridge, CB2 1EW, United \\ Kingdom \\ E-mail: benjamin.rotenberg@upmc.fr
}

\begin{abstract}
An attempt is made to understand the underscreening effect, observed in concentrated electrolyte solutions or melts, on the basis of simple, admittedly crude models involving charged (for the ions) and neutral (for the solvent molecules) hard spheres. The thermodynamic and structural properties of these "primitive" and "semiprimitive" models are calculated within the mean spherical approximation (MSA), which provides the basic input required to determine the partial density response functions. The screening length $\lambda_{S}$, which is unambiguously defined in terms of the wave-number-dependent response functions, exhibits a cross-over from a low density, Debye-like regime, to a regime where $\lambda_{S}$ increases with density beyond a critical density at which the Debye length $\lambda_{D}$ becomes comparable to the ion diameter. In this high density regime the ratio $\lambda_{S} / \lambda_{D}$ increases according to a power law, in qualitative agreement with experimental measurements, albeit at a much slower rate.

PACS numbers:
\end{abstract}

Submitted to: J. Phys.: Condens. Matter - Special Issue "Liquid Matter Conference" 


\section{Introduction}

The theoretical understanding of the microscopic structure and dynamics of ionic solutions and melts, both in the bulk and at interfaces, has made enormous progress since the pioneering work of Gouy [1], Chapman [2], Debye and Hückel [3, 4], Onsager (see [5]) and many others. The "Coulomb singularity", due to the infinite range of Coulombic forces, entails a very specific behaviour, in particular the "screening" mechanism, first examined, under bulk conditions, by Debye and Hückel [3, 4] within a mean-field framework. Rigorous statistical mechanics investigation have led to a wealth of "exact results", including sum rules which provide useful guides for systematic approximation schemes (for an exhaustive review see [6]). The adsorption of ions near a charged surface, first suggested by Stern [7] leads to layering of ions of alternative charge; at high concentration, this layering can entail an "overscreening" phenomenon, whereby successive layers can overshoot local charge neutrality, and can lead to an effective attraction between equally charged parallel surfaces for short separations (for a review, see [8]).

Recently, a number of surface force measurements have revealed a new and unexpected behaviour, namely "underscreening" in ionic melts and concentrated electrolyte solutions. [9, 10, 11] Careful measurements of the force acting between charged mica sheets plunged into an electrolyte show that it decays exponentially with the separation $D$ between sheets, beyond a short-range layering regime. The variation of the screening length $\lambda_{S}$ (characterizing the exponential decay) with ion concentration crosses over from a Debye regime, where $\lambda_{S} \sim \lambda_{D}$, the Debye length which decreases with concentration, to a regime where $\lambda_{S}$ increases with concentration to reach values much larger than $\lambda_{D}$. The cross-over occurs when $\lambda_{D}$ becomes comparable to the mean ion diameter $\sigma$. Moreover, this behaviour is found to be "universal", i.e. the ratio $\lambda_{S} / \lambda_{D}$ obeys a simple scaling, viz:

$$
\begin{array}{rlrl}
\lambda_{S} / \lambda_{D} & =1 & & \\
& =\left(\sigma / \lambda_{D}\right)^{3} & & \lambda_{D}>\sigma \\
& , & \lambda_{D}<\sigma
\end{array}
$$

which holds for a wide range of pure (solvent-free) ionic liquids and of ionic solutions. [11]

A number of ingenious phenomenological interpretations of the scaling relations (1) have been put forward $[11,12,13]$, but in the present paper we adopt a "first principles" approach. We consider primitive and semi-primitive models of ionic solutions, whereby ions are taken to be charged hard spheres, while the solvent is either implicit, providing a dielectric permittivity $\epsilon$ (primitive model), or taken to be neutral hard spheres in addition to providing a given $\epsilon$ (semi-primitive model). The primitive model with $\epsilon=1$ naturally applies to the solvent-free case. These models are admittedly very crude since they neglect, inter alia, the dipole and higher order moments carried by the solvent molecules, as well as the polarizability of the solvent and ions. They have however the advantage that their thermodynamic and structural properties can be readily calculated using standard tools of liquid state theory, in particular the mean- 
spherical approximation (MSA) and its extensions [14], giving unambiguous access to the concentration dependence of the screening length $\lambda_{S}$.

The outline of the paper is the following: In section 2, Landau fluctuation theory is applied to calculate the partial structure factors of the primitive and semiprimitive models as functions of concentration; the required thermodynamic properties are calculated within MSA. In section 3, the Landau approach is reformulated and generalized in terms of wavenumber-dependent response functions. The asymptotic behaviour of the partial structure factors is determined by the poles in the plane of complex wavenumbers $k=k^{\prime}+i k^{\prime \prime}$, along the lines of Ref. [15]. Section 4 presents the MSA-based numerical results for the screening length $\lambda_{S}$ of both primitive and semiprimitive models. Conclusions are drawn in Section 5, together with plans for future work.

\section{Landau fluctuation theory}

The semi-primitive model is a three component hard-sphere system of cations (charge $q_{1}=z_{1} e$, diameter $\sigma_{1}$ ), anions (charge $q_{2}=z_{2} e$, diameter $\sigma_{2}$ ) and solvent molecules (charge $q_{3}=0$, diameter $\left.\sigma_{3}\right)$. The number densities are $\rho_{\alpha}=N_{\alpha} / V(\alpha=1,2,3)$, while global charge neutrality implies: $z_{1} \rho_{1}+z_{2} \rho_{2}=0$. Let:

$$
f\left(\rho_{1}, \rho_{2}, \rho_{3}, T\right)=\frac{F\left(N_{1}, N_{2}, N_{3}, V, T\right)}{V}
$$

be the Helmholtz free energy per unit volume. Similarly one defines the internal energy per unit volume, $u$, and the entropy per unit volume, $s$. The chemical potentials are defined by:

$$
\mu_{\alpha}=\left(\frac{\partial f}{\partial \rho_{\alpha}}\right)_{\rho_{\bar{\alpha}}, T}
$$

while:

$$
\delta s=\frac{1}{T} \delta u-\frac{1}{T} \sum_{\alpha} \mu_{\alpha} \delta \rho_{\alpha} .
$$

Consider local fluctuations of the variables $T$ and $\rho_{\alpha}$ around their equilibrium values, and expand the entropy fluctuation $\delta s(\mathbf{r})$ to second order in $\delta T(\mathbf{r})$ and $\delta \rho_{\alpha}(\mathbf{r})$, along the lines of Landau fluctuation theory [16]:

$$
\begin{aligned}
\delta s(\mathbf{r})= & \frac{1}{T} \delta u(\mathbf{r})-\frac{1}{T} \sum_{\alpha} \mu_{\alpha} \delta \rho_{\alpha}(\mathbf{r}) \\
& -\frac{1}{2}\left\{\frac{c_{V}}{T^{2}}[\delta T(\mathbf{r})]^{2}+\frac{1}{T} \sum_{\alpha} \sum_{\beta}\left(\frac{\partial \mu_{\alpha}}{\partial \rho_{\beta}}\right) \delta \rho_{\alpha}(\mathbf{r}) \delta \rho_{\beta}(\mathbf{r})\right\}
\end{aligned}
$$

where $c_{V}=\left(\frac{\partial u}{\partial T}\right)_{\left\{\rho_{\alpha}\right\}}$ is the specific heat per unit volume.

We temporarily restrict the discussion to the solvent-free case $\left(\rho_{3}=0\right)$ and generalize the calculation of Ref. [17], valid for the one-component plasma (OCP: point ions in a uniform neutralizing background of opposite charge), to the primitive model. 
The local fluctuation in internal energy is the sum of the kinetic energy and electrostatic contributions:

$$
\delta u(\mathbf{r})=u(\mathbf{r})-u_{0}=-\frac{1}{2} \rho_{m}|\mathbf{v}(\mathbf{r})|^{2}-\frac{\epsilon}{8 \pi}|\mathbf{E}(\mathbf{r})|^{2}
$$

where $\mathbf{v}(\mathbf{r})$ is the local fluid velocity, $\rho_{m}=m_{1} \rho_{1}+m_{2} \rho_{2}$ is the global mass density, $\epsilon$ is the dielectric permittivity and $\mathbf{E}(\mathbf{r})$ is the local electric field, which satisfies Poisson's equation:

$$
\nabla \cdot \mathbf{E}(\mathbf{r})=\frac{4 \pi e}{\epsilon}\left[z_{1} \delta \rho_{1}(\mathbf{r})+z_{2} \delta \rho_{2}(\mathbf{r})\right] .
$$

Switching to Fourier space representation, e.g.:

$$
\delta \rho_{\alpha}(\mathbf{r})=\frac{1}{V} \sum_{\mathbf{k}} \rho_{\mathbf{k} \alpha} e^{-i \mathbf{k} \cdot \mathbf{r}}
$$

and integrating both sides of Eq. (5) over the volume $V$, one arrives at:

$$
\begin{aligned}
\frac{S}{k_{B}}= & \frac{1}{k_{B}} \int s(\mathbf{r}) \mathrm{d} \mathbf{r}=\frac{S_{0}}{k_{B}}+\frac{1}{k_{B}} \int \delta s(\mathbf{r}) \mathrm{d} \mathbf{r} \\
= & \frac{S_{0}}{k_{B}}-\frac{\rho_{m}}{2 k_{B} T_{0} V} \sum_{\mathbf{k}}^{\prime} \mathbf{v}_{\mathbf{k}} \cdot \mathbf{v}_{-\mathbf{k}}-\frac{c_{V}^{0}}{2 k_{B} T_{0}^{2} V} \sum_{\mathbf{k}}^{\prime} T_{\mathbf{k}} T_{-\mathbf{k}} \\
& -\frac{1}{2 k_{B} T_{0} V} \sum_{\mathbf{k}}^{\prime} \frac{4 \pi e^{2}}{\epsilon k^{2}}\left[z_{1}^{2}\left|\rho_{\mathbf{k} 1}\right|^{2}+z_{2}^{2}\left|\rho_{\mathbf{k} 2}\right|^{2}+2 z_{1} z_{2} \Re\left(\rho_{\mathbf{k} 1} \rho_{-\mathbf{k} 2}\right)\right] \\
& -\frac{1}{2 k_{B} T_{0} V} \sum_{\mathbf{k}}^{\prime}\left[\chi_{11}^{-1}\left|\rho_{\mathbf{k} 1}\right|^{2}+\chi_{22}^{-1}\left|\rho_{\mathbf{k} 2}\right|^{2}+2 \chi_{12}^{-1} \Re\left(\rho_{\mathbf{k} 1} \rho_{-\mathbf{k} 2}\right)\right]
\end{aligned}
$$

where the subscript 0 refers to equilibrium quantities, $\Re$ refers to the real part of a complex argument, $k_{B}$ is Boltzmann's constant and:

$$
\chi_{\alpha \beta}^{-1}=\left(\frac{\partial \mu_{\alpha}}{\partial \rho_{\beta}}\right)_{0}=\left(\frac{\partial^{2} f}{\partial \rho_{\alpha} \partial \rho_{\beta}}\right)_{0}
$$

The probability of a fluctuation is:

$$
\mathcal{P}\left(\left\{\mathbf{v}_{\mathbf{k}}, T_{\mathbf{k}}, \rho_{\mathbf{k} 1}, \rho_{\mathbf{k} 2}\right\}\right) \sim \exp \left[\frac{\Delta S}{k_{B}}\right] \sim \prod_{\mathbf{k}} \mathcal{P}\left(\mathbf{v}_{\mathbf{k}}, T_{\mathbf{k}}, \rho_{\mathbf{k} 1}, \rho_{\mathbf{k} 2}\right)
$$

with $\Delta S=S-S_{0}$ the associated change in entropy. Eq. (9) shows that the velocity and temperature fluctuations are decoupled from the density fluctuation.

The key objective is the calculation of the charge-charge structure factor:

$$
S_{Z Z}(k)=\frac{1}{V}\left\langle\rho_{\mathbf{k}}^{Z} \rho_{-\mathbf{k}}^{Z}\right\rangle=\frac{\int \mathcal{P}\left(\rho_{\mathbf{k} 1}, \rho_{\mathbf{k} 2}\right) \rho_{\mathbf{k}}^{Z} \rho_{-\mathbf{k}}^{Z} \mathrm{~d} \rho_{\mathbf{k} 1} \mathrm{~d} \rho_{\mathbf{k} 2}}{\int \mathcal{P}\left(\rho_{\mathbf{k} 1}, \rho_{\mathbf{k} 2}\right) \mathrm{d} \rho_{\mathbf{k} 1} \mathrm{~d} \rho_{\mathbf{k} 2}}
$$

A lengthy but straightforward calculation leads to the required expression for the dimensionless charge-charge structure factor:

$$
\frac{S_{Z Z}(k)}{\sum_{\alpha} \rho_{\alpha} z_{\alpha}^{2}}=\frac{\lambda_{D}^{2} k^{2}}{1+\lambda_{S}^{2} k^{2}}
$$


which is valid for all $\mathbf{k}$ at this level of theory (Gaussian fluctuations), but is also a good approximation of more accurate ones in the small $\mathbf{k}$ limit, and where the Debye length is given by:

$$
\lambda_{D}=\left[4 \pi l_{B}\left(\rho_{1} z_{1}^{2}+\rho_{2} z_{2}^{2}\right)\right]^{-1 / 2}
$$

with $l_{B}=e^{2} / \epsilon k_{B} T$ the Bjerrum length, while the screening length $\lambda_{S}$ is defined by:

$$
\lambda_{S}=\left[\frac{1}{4 \pi l_{B}} \frac{\left(\chi_{11}^{-1} \chi_{22}^{-1}-\chi_{12}^{-2}\right)}{\left(Z_{1}+Z_{2}\right)^{2}} \frac{\rho_{0}^{2} \chi_{T}}{k_{B} T_{0}}\right]^{1 / 2}
$$

where $Z_{\alpha}=\left|z_{\alpha}\right|$ and $\chi_{T}$ is the isothermal compressibility $\chi_{T}=-\frac{1}{V}\left(\frac{\partial V}{\partial P}\right)_{T}$, i.e.:

$$
\chi_{T}^{-1}=\rho_{0}^{2}\left(\frac{\partial^{2} f}{\partial \rho_{0}^{2}}\right)_{T}=\frac{\rho_{0}^{2}}{\left(Z_{1}+Z_{2}\right)^{2}}\left[Z_{2}^{2} \chi_{11}^{-1}+Z_{1}^{2} \chi_{22}^{-1}+2 Z_{1} Z_{2} \chi_{12}^{-1}\right] .
$$

Note that $\lambda_{S}^{2}$ can be expressed as the ratio $-I_{4} / I_{2}$ where $I_{2}$ and $I_{4}$ are the coefficients of $k^{2}$ and $k^{4}$ terms in the small $k$ expansion of $S_{Z Z}(k)$; these coefficients have been derived from second and fourth moment sum rules within linear response theory [18] and lead back to the result (15).

$\lambda_{S}$ can now be estimated by calculating $\chi_{T}$ and the $\chi_{\alpha \beta}^{-1}$, defined by Eq. (10), using the MSA expression for the free energy of the primitive model both for the symmetric case $\left(\sigma_{1}=\sigma_{2}, z_{1}=-z_{2}\right)$ [19] and for asymmetric cases [20, 21]. Results for the symmetric case will be presented in Section 4. The main conclusion is that fluctuation theory, using the thermodynamic functions $\chi_{\alpha \beta}^{-1}$ corresponding to the MSA free energy, predicts a screening length $\lambda_{S}$ which is systematically smaller than the Debye length $\lambda_{D}$, and hence cannot account for underscreening.

For the sake of completeness, we also quote the fluctuation theory results for the number-charge and number-number structure factors $S_{N Z}(k)$ and $S_{N N}(k)$ :

$$
S_{N Z}(k)=\frac{1}{V}\left\langle\rho_{\mathbf{k} N} \rho_{-\mathbf{k} Z}\right\rangle=\rho_{0} \frac{\lambda_{N Z}^{2} k^{2}}{1+\lambda_{S}^{2} k^{2}}
$$

where $\rho_{0}=\rho_{1}+\rho_{2}=\frac{N}{V}$ and:

$$
\lambda_{N Z}^{2}=\frac{\rho_{0} \chi_{T}}{4 \pi l_{B}} \frac{z_{2} \chi_{11}^{-1}+z_{1} \chi_{22}^{-1}-\left(z_{1}+z_{2}\right) \chi_{12}^{-1}}{\left(Z_{1}+Z_{2}\right)^{2}} .
$$

As expected $S_{N Z}(k)=0$ in the fully symmetric case $\left(z_{1}=-z_{2}\right.$ and $\chi_{11}^{-1}=\chi_{22}^{-1}$, i.e. charge and number densities are uncorrelated). Note also that $\lim _{k \rightarrow 0} S_{N Z}(k)=0$, as is the case for $S_{Z Z}(k)$. Finally:

$$
S_{N N}(k)=\frac{1}{V}\left\langle\rho_{\mathbf{k} N} \rho_{-\mathbf{k} N}\right\rangle=\frac{\rho_{0}^{2} k_{B} T_{0} \chi_{T}\left[1+\lambda_{N}^{2} k^{2}\right]}{1+\lambda_{S}^{2} k^{2}}
$$

where:

$$
\lambda_{N}^{2}=\frac{1}{4 \pi l_{B} k_{B} T_{0}} \frac{\chi_{11}^{-1}+\chi_{22}^{-1}-2 \chi_{12}^{-1}}{\left(Z_{1}+Z_{2}\right)^{2}} .
$$

As expected, $\lim _{k \rightarrow 0} S_{N N}(k)=\rho_{0}^{2} k_{B} T_{0} \chi_{T}=\rho_{0} \frac{\chi_{T}}{\chi_{T}^{i d}}$. Note that the expressions (13), (17) and (19) of the structure factors imply that the corresponding correlation functions $h_{Z Z}(r), h_{N Z}(r)$ and $h_{N N}(r)$ ultimately decay on the same length scale, as expected [15]. 


\section{Screening in terms of partial density response functions}

The failure of fluctuation theory, which is only adapted to the $k \rightarrow 0$ limit, suggests that finite wavevectors $\mathbf{k}$ must be included in a realistic description of screening incorporating excluded volume effects, as suggested by the expreimental evidence of a cross-over in screening behaviour when $k \sigma \approx 1$. [11] We hence reformulate the theory in terms of $k$-dependent partial response functions $\hat{\chi}_{\alpha \beta}$, defined by:

$$
\hat{\chi}_{\alpha \beta}^{-1}(k)=\frac{\delta_{\alpha \beta}}{\rho_{\alpha}}-\hat{c}_{\alpha \beta}(k)
$$

which occur naturallly in the description of the linear response to an external field [14];

the $\hat{c}_{\alpha \beta}(k)$ are the Fourier transforms of the direct correlation functions $c_{\alpha \beta}(r)$. The relations between the $\hat{\chi}_{\alpha \beta}^{-1}(k)$ and the partial structure factors:

$$
S_{\alpha \beta}(k)=\frac{1}{V}\left\langle\rho_{\mathbf{k} \alpha} \rho_{-\mathbf{k} \beta}\right\rangle=\rho_{\alpha} \delta_{\alpha \beta}+\rho_{\alpha} \rho_{\beta} \hat{h}_{\alpha \beta}(k)
$$

where $\hat{h}_{\alpha \beta}(k)$ are the Fourier transforms of the pair correlation functions, are easily established via the Ornstein-Zernike (OZ) relations between the $\hat{c}_{\alpha \beta}(k)$ and the $\hat{h}_{\alpha \beta}(k)$. [14]

Restricting once more the subsequent calculations to the primitive (i.e. solventfree) model, the coupled Orstein-Zernike equations lead directly to the following matrix relation between the $S_{\alpha \beta}(k)$ and the $\hat{\chi}_{\alpha \beta}^{-1}(k)$ :

$$
\left(\begin{array}{cc}
S_{11} & S_{12} \\
S_{21} & S_{22}
\end{array}\right)=\frac{1}{\hat{\chi}_{11}^{-1} \hat{\chi}_{22}^{-1}-\hat{\chi}_{12}^{-1} \hat{\chi}_{21}^{-1}}\left(\begin{array}{cc}
\hat{\chi}_{22}^{-1} & -\hat{\chi}_{21}^{-1} \\
-\hat{\chi}_{12}^{-1} & \hat{\chi}_{11}^{-1}
\end{array}\right)
$$

and hence:

$$
S_{Z Z}(k)=\sum_{\alpha} \sum_{\beta} z_{\alpha} z_{\beta} S_{\alpha \beta}(k)=\frac{z_{1}^{2} \hat{\chi}_{22}^{-1}(k)+z_{2}^{2} \hat{\chi}_{11}^{-1}(k)-2 z_{1} z_{2} \hat{\chi}_{12}^{-1}(k)}{\hat{\chi}_{11}^{-1}(k) \hat{\chi}_{22}^{-1}(k)-\hat{\chi}_{12}^{-1}(k) \hat{\chi}_{21}^{-1}(k)}
$$

Note that the $k \rightarrow 0$ limit of $\hat{\chi}_{\alpha \beta}^{-1}(k)$ is related to the thermodynamic derivatives defined in Eq. (10), thus pointing to the link between the fluctuation theory of Section 2 and the present extension to finite $k$. In charged systems, the $\hat{c}_{\alpha \beta}(k)$ split into a Coulombic and short-range contribution:

$$
\hat{c}_{\alpha \beta}(k)=-\frac{4 \pi l_{B}}{k^{2}} z_{\alpha} z_{\beta}+\hat{c}_{\alpha \beta}^{S}(k)
$$

Using the definition (21) and substituting into (24), one arrives at the final expression for $S_{Z Z}(k)$ :

$$
\begin{gathered}
S_{Z Z}(k)=\frac{k^{2} A(k)}{k^{2}+4 \pi l_{B} A(k)} \\
A(k)=\frac{\rho_{1} z_{1}^{2}\left[1-\rho_{2} \hat{c}_{22}^{S}(k)\right]+\rho_{2} z_{2}^{2}\left[1-\rho_{1} \hat{c}_{11}^{S}(k)\right]+2 \rho_{1} \rho_{2} z_{1} z_{2} \hat{c}_{12}^{S}(k)}{\left[1-\rho_{1} \hat{c}_{11}^{S}(k)\right]\left[1-\rho_{2} \hat{c}_{22}^{S}(k)\right]-\rho_{1} \rho_{2}\left[\hat{c}_{12}^{S}(k)\right]^{2}}
\end{gathered}
$$


Since the $c_{\alpha \beta}^{S}(r)$ are short-ranged functions, one may expand their Fourier transforms, as well as the resulting $A(k)$, into even powers of $k[22]$ :

$$
\begin{aligned}
& \hat{c}_{\alpha \beta}^{S}(k)=c_{\alpha \beta}^{(0)}+c_{\alpha \beta}^{(2)} k^{2}+\mathcal{O}\left(k^{4}\right) \\
& A(k)=A^{(0)}+A^{(2)} k^{2}+\mathcal{O}\left(k^{4}\right)
\end{aligned}
$$

where:

$$
A^{(0)}=\frac{\rho_{1} z_{1}^{2}\left[1-\rho_{2} c_{22}^{(0)}\right]+\rho_{2} z_{2}^{2}\left[1-\rho_{1} c_{11}^{(0)}\right]+2 \rho_{1} \rho_{2} z_{1} z_{2} c_{12}^{(0)}}{\left[1-\rho_{1} c_{11}^{(0)}\right]\left[1-\rho_{2} c_{22}^{(0)}\right]-\rho_{1} \rho_{2}\left[c_{12}^{(0)}\right]^{2}}
$$

The corresponding small $k$ expansion of the charge-charge structure factor is:

$$
\frac{S_{Z Z}(k)}{\sum_{\alpha} \rho_{\alpha} z_{\alpha}^{2}}=k^{2} \lambda_{D}^{2}-\frac{k^{4} \lambda_{D}^{2}}{4 \pi l_{B} A^{(0)}}
$$

Minus the ratio of the coefficients of $k^{4}$ and $k^{2}$ leads back to an expression of $\lambda_{S}^{2}$ identical to that provided by Eq. (15), as expected. The thermodynamic derivatives $\chi_{\alpha \beta}^{-1}=k_{B} T \hat{\chi}_{\alpha \beta}^{-1}(k=0)=k_{B} T\left[\frac{\delta_{\alpha \beta}}{\rho_{\alpha}}-c_{\alpha \beta}^{(0)}\right]$ can be calculated analytically in the symmetric case within the MSA, with the result for the screening length:

$$
\left(\lambda_{S}^{M S A}\right)^{2}=\left[1-2 \Gamma^{2} \sigma^{2}\left(1+\frac{2}{3} \Gamma \sigma+\frac{1}{6} \Gamma^{2} \sigma^{2}\right)\right] \lambda_{D}^{2}
$$

where $\Gamma^{-1}=\lambda_{D}\left[1+\sqrt{1+2 \sigma / \lambda_{D}}\right]$. The result (30) is similar, although not identical to an expression derived by Attard [23] based on second moment considerations. As pointed out in Section 2, the resulting $\lambda_{S}^{M S A}$ are always less than $\lambda_{D}$. To make further progress we must return to the exact expression (26) for $S_{Z Z}(k)$, and exploit the full $k$-dependence of the response function $\hat{\chi}_{\alpha \beta}^{-1}(k)$, or equivalently of the $\hat{c}_{\alpha \beta}^{S}(k)$.

The long-range behaviour of the charge density correlation function:

$$
h_{Z Z}(r)=\sum_{\alpha} \sum_{\beta} z_{\alpha} z_{\beta}\left[\rho_{\alpha} \delta_{\alpha \beta}+\rho_{\alpha} \rho_{\beta} h_{\alpha \beta}(r)\right]
$$

is determined by the poles of its Fourier transform $S_{Z Z}(k)$ in the complex wavenumbers $k=k^{\prime}+i k^{\prime \prime}$, i.e. by the zeros of the denominator: $\mathcal{D}(k)=k^{2}+4 \pi l_{B} A(k)$ in Eq. (26). [15] The dominant (longest) decay length is controlled by the pole of $S_{Z Z}(k)$ closest to the real axis in the complex wavenumber plane. If this pole lies on the imaginary axis $k^{\prime \prime}$, as occurs at low enough concentrations, the decay of $h_{Z Z}(r)$ is purely exponential, while for poles with a non-zero real part $k^{\prime}$, the charge density correlations exhibit an oscillatory behaviour, at least at short distances $r$ between ions, due to layering (for details, see Ref. [15]). The complex roots of $\mathcal{D}(k)$ are determined numerically and the key results of this numerical analysis are summarized in Section 4.

Before discussing our results for the bulk correlation function $h_{Z Z}(r)$, it is important to recall why these results are relevant for the interpretation of the experimental data which apply to a liquid film confined between two surfaces. The key reason is that the asymptotic decay of the density profile of such an inhomogeneous fluid is governed by a correlation (in this case screening) length which is identical to that characterizing the static correlations in a bulk fluid at a given density and temperature [24, 25]. 


\section{MSA results for underscreening at high concentrations}

Some of the numerical results based on the expressions derived in Sections 2 and 3 for the solvent-free primitive model and for the three-component semi-primitive model are summarized in Figures 1 to 3. In all cases examined so far, the ion and solvent diameters are equal $\left(\sigma_{1}=\sigma_{2}=\sigma_{3}=\sigma\right)$, while cations and anions carry opposite charges $\left(z_{1}=-z_{2}\right)$. The diameter and valencies are chosen with the case of sodium chloride $(\mathrm{NaCl})$ in mind, namely $\sigma=0.3 \mathrm{~nm}$ and $z_{1,2}= \pm 1$.

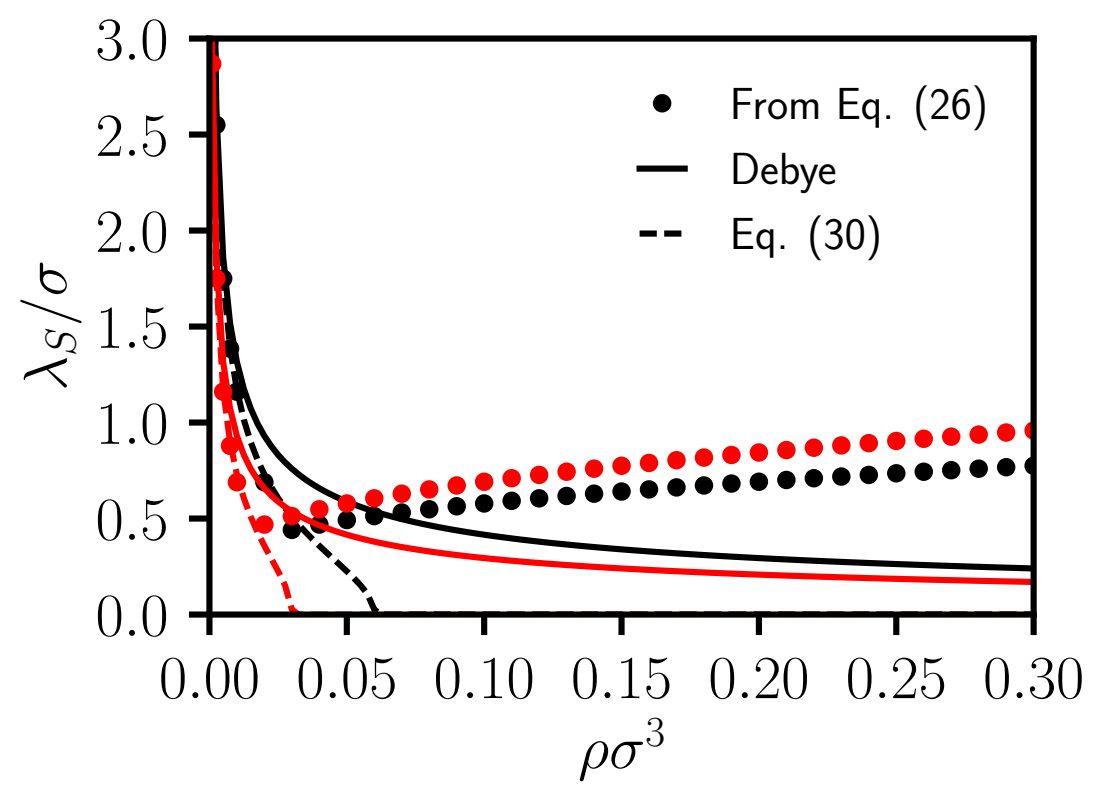

Figure 1. Reduced screening length $\lambda_{S} / \sigma$ as a function of the reduced density $\rho \sigma^{3}$, for two values of the reduced Bjerrum length $l_{B} / \sigma=e^{2} / \epsilon k_{B} T \sigma$. The solid lines correspond to the Debye prediction $\lambda_{S}=\lambda_{D}$, while the dashed lines are the Landau fluctuation theory results (30) using the MSA predictions for the thermodynamic quantities $\chi_{\alpha \beta}^{-1}$. The symbols are calculated from the complex poles of the structure factor $S_{Z Z}(k)$, Eq. (26), using once more the MSA for the calculation of the $k$-depedent $\hat{c}_{\alpha \beta}^{S}(k)$. Calculations correspond to ion diameter $\sigma=3 \AA$ and charges $\pm e$, in water at room temperature $(\epsilon=78, T=300 \mathrm{~K})$ for $l_{B} / \sigma=2.3$ (black symbols), while the value 4.6 corresponds to $\epsilon=39$ (red symbols).

Figure 1 shows the variation of the reduced screening length $\lambda_{S} / \sigma$ vs the reduced density $\rho \sigma^{3}$, for two values of the reduced Bjerrum length $l_{B} / \sigma=e^{2} / \epsilon k_{B} T \sigma$. For the above-mentioned case of $\mathrm{NaCl}$, the value $l_{B} / \sigma=2.3$ corresponds to room temperature water conditions $(\epsilon=78, T=300 \mathrm{~K})$, while the value 4.6 corresponds to $\epsilon=39$. The continuous curves correspond to the Debye prediction $\lambda_{S}=\lambda_{D}$, while the dashed curves are the Landau fluctuation theory results (30) using the MSA predictions for the thermodynamic quantities $\chi_{\alpha \beta}^{-1}$. The symbols are calculated from the complex poles of the structure factor $S_{Z Z}(k)$, Eq. (26), using once more the MSA for the calculation of 
the $k$-depedent $\hat{c}_{\alpha \beta}^{S}(k)$.

While $\lambda_{D}$ decreases steadily like $1 / \sqrt{\rho \sigma^{3}}$, the Landau fluctuation theory, which applies only to small $k$, predicts that $\lambda_{S}$ decreases much faster and goes to zero when $\rho \sigma^{3} \approx 0.03$ (corresponding approximately to $2 \mathrm{~mol} / \mathrm{L}$ with the above parameters) under water at room temperature conditions, and at $\rho \sigma^{3} \approx 0.06$ for the lower $\epsilon$. On the other hand, the MSA predictions within the density response formalism of Section 3 point to a clear-cut cross-over behaviour from a rapid drop of $\lambda_{S}$ with density, close to the predictions of Landau fluctuation theory, up to $\rho \sigma^{3} \approx 0.02\left(l_{B} / \sigma=2.3\right)$ or $\rho \sigma^{3} \approx 0.03$ $\left(l_{B} / \sigma=4.6\right)$, followed by an increase of $\lambda_{S}$ beyond those cross-over densities. This cross-over corresponds precisely to the underscreening observed experimentally at high densities or concentrations [11], even though the order of magnitude of $\lambda_{S}$ remains here comparable to the ion diameter in this concentration regime. Note that the minimum of $\lambda_{S}$ vs $\rho \sigma^{3}$ is in fact a cusp, due to the cross-over from a pure exponential to a damped oscillatory decay of the correlations [15]. In the vicinity of the cusp (which occurs at $\sigma / \lambda_{D} \approx 1.228$ within the MSA [15]), the wavelength of the oscillations becomes very long and hence practically unobservable, which explains why no oscillations are observed in the force measured in that range [11].

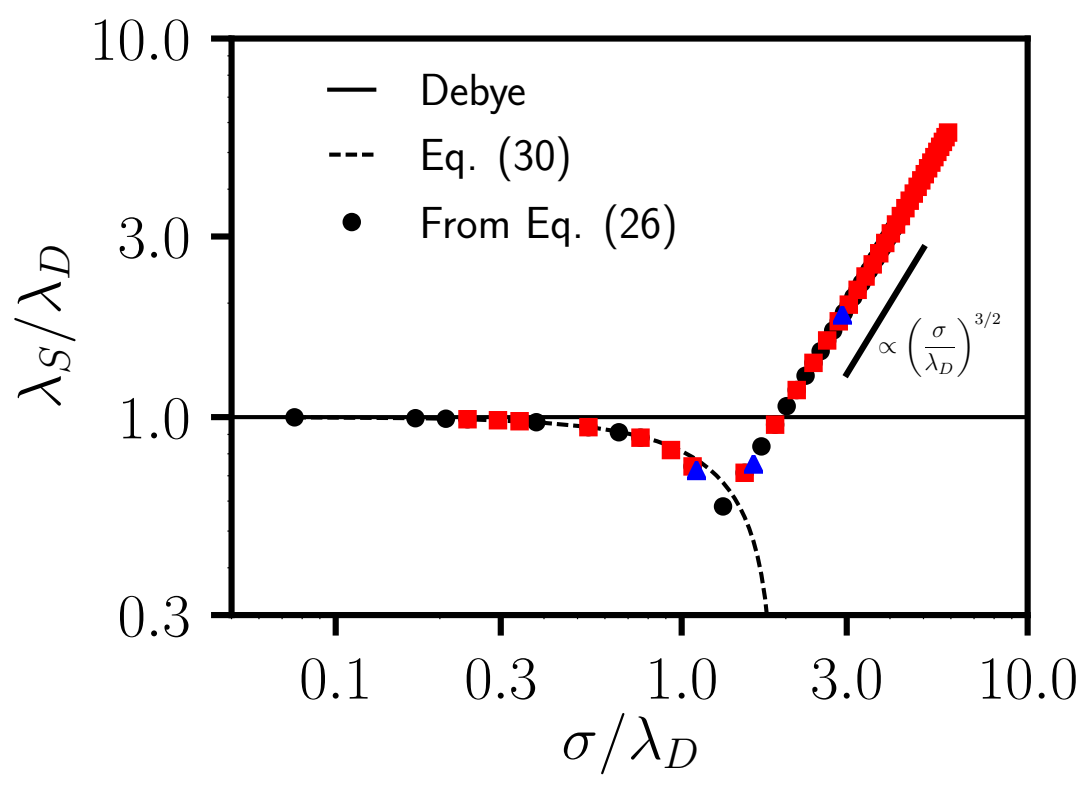

Figure 2. Ratio of the screening length over the Debye length as a function of the ratio of the ion diameter over the Debye length. The horizontal line corresponds to the Debye prediction $\lambda_{S}=\lambda_{D}$, while the dashed line is the Landau fluctuation theory prediction (30) using the MSA results for the thermodynamic quantities $\chi_{\alpha \beta}^{-1}$. The symbols are calculated from the complex poles of the structure factor $S_{Z Z}(k)$, Eq. (26), using once more the MSA for the calculation of the $k$-depedent $\hat{c}_{\alpha \beta}^{S}(k)$. The results correspond to the same parameters as in Figure $1(\bullet,-\square)$ as well as upon changing the permittivity of the solvent according to the experimental values $(\mathbf{\Lambda})$ reported in Ref. $[10,11,12]$. 
To make contact with the experimental scaling relation (1) $[10,11,12]$, we plot the ratio $\lambda_{S} / \lambda_{D}$ vs $\sigma / \lambda_{D}$ on a log-log scale in Figure 2, for the solvent-free (primitive model) case, once again for the two values of $l_{B} / \sigma$, namely 2.3 and 4.6. We also indicate on this plot the predictions of the same theory when changing the permittivity of the medium upon increasing concentration according to the experimental values reported in Ref. $[10,11,12]$. The Landau fluctuation theory results drop rapidly below the Debye prediction $\lambda_{S} / \lambda_{D}=1$ when $\sigma / \lambda_{D}$ approaches 1 . The predictions based on the density response formalism, Eq. (26), using MSA results for $\hat{c}_{\alpha \beta}^{S}(k)$, again clearly indicate a sharp cross-over from the Landau fluctuation prediction, at $\sigma / \lambda_{D}$ close to (and slightly above) 1 towards a rapid increase for $\sigma / \lambda_{D}>1$, in qualitative agreement with experimental data. As emphasized in Ref. [15], the MSA necessarily leads to a "universal" scaling relation (at least for fixed permittivity $\epsilon$ ), as illustrated in Fig. 2 where the black and red dots fall on the same straight line. More unexpectedly, even when $\epsilon$ is allowed to vary with concentration (triangles in Fig. 2), the results obey the same scaling. The slope of the log-log plot in Figure 2 suggests a scaling $\lambda_{S} / \lambda_{D} \propto\left(\sigma / \lambda_{D}\right)^{\nu}$ with $\nu=3 / 2$ which is well below the value $\nu=3$ suggested by the experimental data [12], i.e. the theoretical screening length increases much less rapidly with density than observed experimentally. In other words, underscreening is indeed predicted by our theory, but is less dramatic than suggested by the experimental data. Note also the dip in $\lambda_{S} / \lambda_{D}=1$ predicted just before the cross-over density, which is not observed in the experimental measurements.

Finally, Figure 3 shows a similar $\lambda_{S} / \lambda_{D}$ vs $\sigma / \lambda_{D}$ plot, comparing results for the two-component primitive model and the three-component semi-primitive model with a hard sphere solvent of diameter equal to that of the ions. For the latter, the total volume fraction $\eta=\frac{\pi}{6}\left(\rho_{1}+\rho_{2}+\rho_{3}\right) \sigma^{3}$ is held fixed at a value $\eta=0.47$, which corresponds approximately to that of liquid water for a diameter $\sigma=0.3 \mathrm{~nm}$, meaning that the ion concentration increases, while the solvent concentration decreases, as $\sigma / \lambda_{D}$ increases. In the latter case the screening length is computed as the imaginary part of the pole of $S_{Z Z}(k)$ closest to the real axis, determined numerically from the roots of the denominator:

$$
\begin{gathered}
\mathcal{D}_{3}(k)=k^{2}\left[\left(\left[1-\rho_{1} \hat{c}_{11}(k)\right]\left[1-\rho_{2} \hat{c}_{22}(k)\right]-\rho_{1} \rho_{2}\left[\hat{c}_{12}(k)\right]^{2}\right)\left[1-\rho_{3} \hat{c}_{33}(k)\right]\right. \\
\quad-\rho_{1} \rho_{3}\left[\hat{c}_{13}(k)\right]^{2}\left[1-\rho_{2} \hat{c}_{22}(k)\right]-\rho_{2} \rho_{3}\left[\hat{c}_{23}(k)\right]^{2}\left[1-\rho_{1} \hat{c}_{11}(k)\right] \\
\left.-2 \rho_{1} \rho_{2} \rho_{3} \hat{c}_{12}(k) \hat{c}_{23}(k) \hat{c}_{31}(k)\right]
\end{gathered}
$$

In both cases, the cross-over occurs as already shown in Figure 2 for the primitive model, but the scaling exponent $\nu$ is smaller, close to $\nu=1$, in the case of the semi-primitive model. Even though this exponent is further from the experimental one compared to the primitive model case, the present calculations based on simple models of ionic solutions confirm that the cross-over behaviour and subsequent underscreening regime are driven by excluded volume effects which become predominant for $\lambda_{D} \lesssim \sigma$. However improvements of the microscopic model are required to explain the scaling law and large screening length observed experimentally. 


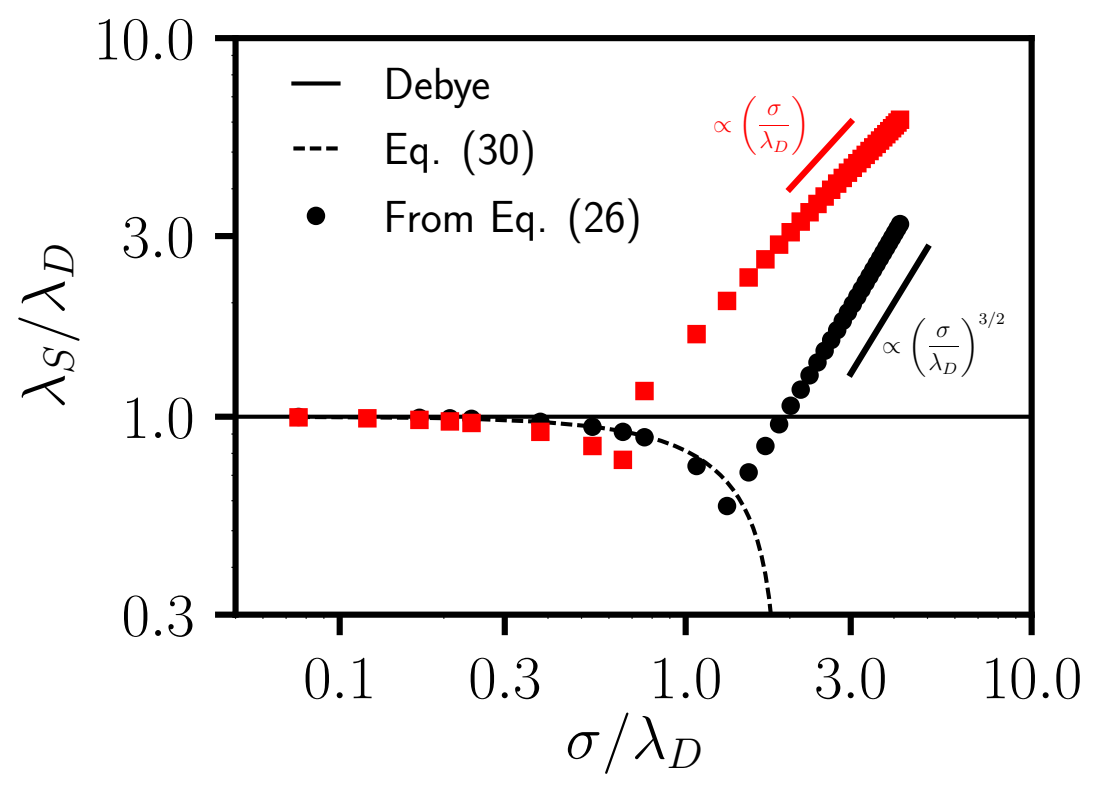

Figure 3. Ratio between the screening length and the Debye screening length as a function of the ratio between the ion diameter and the Debye screening length, for $l_{B} / \sigma=2.3$. The solid lines correspond to the Debye prediction $\lambda_{S}=\lambda_{D}$, while the dashed lines are the Landau fluctuation theory results (30) using the MSA predictions for the thermodynamic quantities $\chi_{\alpha \beta}^{-1}$. The symbols are calculated from the complex poles of the structure factor $S_{Z Z}(k)$ for the two-component primitive model $(\bullet)$ and the three component semi-primitive model $(\mathbf{a})$.

\section{Conclusion}

We have examined the recently discovered underscreening phenomenon in concentrated ionic solutions and melts [11] by considering simple models involving charged and/or neutral hard spheres, namely the so-called primitive and semi-primitive models. The charge-charge structure factor $S_{Z Z}(k)$ was calculated within Landau's gaussian fluctuation theory, appropriate for small wavenumbers $k$, and within the density response function formalism which extends the validity of the Landau fluctuation theory to arbitrary $k$. The required thermodynamic quantities and structural properties of the models were evaluated over a range of concentrations, using the MSA approximation, which is reasonably accurate over a wide range of hard sphere packing fractions. The hard sphere diameter used in the MSA calculations is an effective diameter taking into account the "softness" of the true ion-ion interactions; this effective diameter is (weakly) density-dependent [14], so that the packing fraction itself should be regarded as statedependent in more realistic calculations.

Our calculations are capable of reproducing, at least qualitatively, the cross-over from a low concentration regime, where the screening length $\lambda_{S}$ remains close to the 
Debye length $\lambda_{D}$, to a high concentration regime, where $\lambda_{S}$ increases with concentration, contrary to $\lambda_{D}$, thus confirming the underscreening phenomenon. In that regime the growth of $\lambda_{S} / \lambda_{D}$ obeys a simple scaling relation similar to that observed experimentally (see Eq. (1)), but with an exponent $\nu$ which is considerably smaller $(\nu \approx 3 / 2$ compared to $\nu=3$ ); in other words underscreening is underestimated.

This situation is most likely due to the crude representation of the solvent, which is either implicit (primitive model) or reduced to neutral hard spheres (semi-primitive model). Clearly a "civilized" model of the solvent is required, whereby the solvent hard spheres carry an electric dipole and higher multipoles. Analytic solutions of the MSA for dipolar hard spheres are available [26, 27], although the dielectric properties calculated from the MSA approximation become highly unreliable for high dipole moments. The polarizability of solvent molecules and ions also play an important role in realistic descriptions of ionic solutions [28], which is totally absent in the oversimplified models considered in the present work. In future work we plan to generalize the present analysis of underscreening to asymmetric cases, involving unequal particle diameters $\sigma_{1} \neq \sigma_{2} \neq \sigma_{3}$ and ion valences $\left(Z_{1} \neq Z_{2}\right)$ to investigate the effect of these asymmetries on the scaling relations discussed in Section 4 and illustrated in Figures 2 and 3. It is finally worth stressing that the present calculation is for bulk electrolytes. In the experimental set-up, the electrolyte is confined between parallel charged planes. It is conceivable that the charge fluctuations are affected by the confinement and could lead

to a classical Casimir effect, well known in the theory of critical fluctuations (for a review, see [29]).

\section{Acknowledgments}

The authors are grateful to Susan Perkin, Alpha Lee and Bob Evans for helpful discussions.

[1] M. Gouy. Sur la constitution de la charge électrique à la surface d'un électrolyte. Journal de Physique Théorique et Appliquée, 9(1):457-468, 1910.

[2] D. L. Chapman. A contribution to the theory of electrocapillarity. Philosophical Magazine, 25(148):475-481, 1913.

[3] P. Debye and E. Hückel. The theory of electrolytes I. The lowering of the freezing point and related occurrences. Physikalische Zeitschfrift, 24:185-206, 1923.

[4] P. Debye and E. Hückel. The theory of electrolytes II. The border law for electrical conductivity. Physikalische Zeitschfrift, 24:305-325, 1923.

[5] P.C. Hemmer, H. Holden, and S. Kjelstrup, editors. The collected works of Lars Onsager. World Scientific, Singapore, 1996.

[6] Ph. A. Martin. Sum rules in charged fluids. Reviews of Modern Physics, 60(4):1075-1127, 1988.

[7] O. Stern. Zur Theorie Der Elektrolytischen Doppelschicht. Zeitschrift für Elektrochemie und angewandte physikalische Chemie, 30(21-22):508-516, 1924.

[8] J.-P. Hansen and H. Löwen. Effective Interactions Between Electric Double Layers. Annual Review of Physical Chemistry, 51(1):209-242, 2000. 
[9] M. A. Gebbie, H. A. Dobbs, M. Valtiner, and J. N. Israelachvili. Long-range electrostatic screening in ionic liquids. Proceedings of the National Academy of Sciences, 112(24):7432-7437, 2015.

[10] A. M. Smith, A. A. Lee, and S. Perkin. The Electrostatic Screening Length in Concentrated Electrolytes Increases with Concentration. The Journal of Physical Chemistry Letters, 7(12):2157-2163, 2016.

[11] A. A. Lee, C. S. Perez-Martinez, A. M. Smith, and S. Perkin. Underscreening in concentrated electrolytes. Faraday Discussions, 199:239-259, 2017.

[12] A. A. Lee, C. S. Perez-Martinez, A. M. Smith, and S. Perkin. Scaling Analysis of the Screening Length in Concentrated Electrolytes. Physical Review Letters, 119(2), 2017.

[13] Zachary A. H. Goodwin and Alexei A. Kornyshev. Underscreening, overscreening and double-layer capacitance. Electrochemistry Communications, 82(Supplement C):129-133, September 2017.

[14] J. P. Hansen and I. R. McDonald. Theory of Simple Liquids. Elsevier, Amsterdam, 4th edition, 2013.

[15] R.J.F. Leote de Carvalho and R. Evans. The decay of correlations in ionic fluids. Molecular Physics, 83(4):619-654, 1994.

[16] L. D. Landau and E. M. Lifshitz. Statistical Physics. Elsevier, Oxford, 3rd edition, 1980.

[17] P. Vieillefosse and J. P. Hansen. Statistical mechanics of dense ionized matter. V. Hydrodynamic limit and transport coefficients of the classical one-component plasma. Physical Review A, 12(3):1106-1116, 1975.

[18] A. Alastuey and R. Fantoni. Fourth Moment Sum Rule for the Charge Correlations of a TwoComponent Classical Plasma. Journal of Statistical Physics, 163(4):887-913, 2016.

[19] E. Waisman and J. L. Lebowitz. Mean spherical model integral equation for charged hard spheres I. Method of solution. The Journal of Chemical Physics, 56(6):3086-3093, 1972.

[20] L. Blum. Mean spherical model for asymmetric electrolytes. Molecular Physics, 30(5):1529-1535, 1975.

[21] L. Blum and J. S. Høye. Mean spherical model for asymmetric electrolytes. 2. Thermodynamic properties and the pair correlation function. The Journal of Physical Chemistry, 81(13):13111316, 1977.

[22] R. Evans and T. J. Sluckin. A density functional theory for inhomogeneous charged fluids. Molecular Physics, 40(2):413-435, 1980.

[23] Phil Attard. Asymptotic analysis of primitive model electrolytes and the electrical double layer. Physical Review E, 48(5):3604, 1993.

[24] J. R. Henderson and Z. A. Sabeur. Liquid-state integral equations at high density: On the mathematical origin of infinite-range oscillatory solutions. The Journal of Chemical Physics, 97(9):6750-6758, 1992.

[25] R. Evans, J.R. Henderson, D.C. Hoyle, A.O. Parry, and Z.A. Sabeur. Asymptotic decay of liquid structure: oscillatory liquid-vapour density profiles and the Fisher-Widom line. Molecular Physics, 80(4):755-775, 1993.

[26] M. S. Wertheim. Exact Solution of the Mean Spherical Model for Fluids of Hard Spheres with Permanent Electric Dipole Moments. The Journal of Chemical Physics, 55(9):4291-4298, 1971.

[27] G. Stell, G. N. Patey, and J. S. Høye. Dielectric Constants of Fluid Models: Statistical Mechanical Theory and its Quantitative Implementation, pages 183-328. John Wiley \& Sons, Inc., 1981.

[28] S. Tazi, J. J. Molina, B. Rotenberg, P. Turq, R. Vuilleumier, and M. Salanne. A transferable ab initio based force field for aqueous ions. The Journal of Chemical Physics, 136(11):114507, 2012.

[29] A. Gambassi. The Casimir effect: From quantum to critical fluctuations. Journal of Physics: Conference Series, 161(1):012037, 2009. 